\title{
INTRODUCTION D'ESPÈCES DANS LES MILIEUX AQUATIQUES D'EAU DOUCE : LES IMPACTS GÉNÉTIQUES.
}

\author{
P. BERREBI \\ Laboratoire Génome et Populations, UPR 9060 du CNRS, Université Montpellier II, \\ place E. Bataillon, 34095 Montpellier Cedex 05, France.
}

\section{RÉSUMÉ}

Les introductions ont des conséquences aux niveaux écologique, pathologique et génétique. Les conséquences génétiques n'apparaissent que si le taxon introduit s'hybride avec un taxon résident et si leurs descendants ( $F 1)$ sont fertiles. Dans cette revue, les divers types d'interactions génétiques sont classés et de nombreux exemples, non limités à la France, les illustrent.

A la lumière de ces cas concrets, les risques génétiques des introductions sont évalués. Puis deux recommandations, directement liées à ces risques, sont proposées. Selon la première, il est recommandé que le taxon, qu'il soit spécifique ou sous-spécifique, soit l'entité à prendre en compte et non l'espèce. La seconde recommandation s'intéresse aux introductions de taxons pour lesquelles aucune connaissance génétique préalable n'est disponible. Pour éviter les risques génétiques développés dans le texte, il conviendrait d'appliquer un " principe de précaution " selon lequel seules les introductions ou transferts d'une rivière à l'autre d'un même bassin hydrographique seraient acceptables sans contrôle.

Mots-clés : génétique, introduction, repeuplement, introgression, conservation.

\section{SPECIES INTRODUCTION IN FRESHWATER ENVIRONMENTS : GENETIC IMPACTS.}

\section{ABSTRACT}

Species introductions have three kinds of consequences that were discussed during the congress "Species introductions ", Paris February 1996 : consequences in ecology, pathology, and genetics. The latter only occurs when an introduced taxon hybridises with a resident taxon and if their offspring (F1) is fertile.

In this paper, the different types of interactions are classified and illustrated by examples that are not limited to France. Type $0=$ no interaction; type $1=$ non-viable hybrids; type $2=$ production of sterile $F 1$; type 3 = production of weakly fertile F1; type 4 = production of highly fertile $F 1$ and easy backcrosses that can lead to the homogenisation of the two taxa.

For the population, the main genetic risk when hybridisation occurs is an outbreeding depression, which can be explained by the breakdown of coadapted gene complexes and/or the breakdown of local adaptation. For the taxon, the risk is a loss of genetic diversity at the population level and/or at the species (between-population) level.

Altogether, genetic introgression is considered nearly irreversible, and the final consequence can be the extinction of the taxon from a genetic point of view. 
To avoid such a dramatic outcome, some precautions may be proposed :

- several examples developed in the text clearly explain that the specific level is not always the good one for investigation. The subspecific level must often be taken into account. However, a good genetic knowledge is necessary for handling that level of organisation because morphology is generally inefficient in distinguishing local forms. So a preliminary genetic description of the taxa to be introduced is encouraged;

- when no genetic information on the species to be introduced is available, which is almost the general case, precaution must be accepted as a matter of principle. The principle especially adapted to freshwater organisms is to allow introductions, in the absence of genetic knowledge, only if the introduced individuals are taken from the same catchment.

Key-words : genetics, introduction, stocking, introgression, conservation.

\section{INTRODUCTION}

Les introductions d'espèces dans le milieu aquatique français ont fait l'objet d'un colloque réuni à Paris en février 1996. Ce vaste tour d'horizon des cas connus, incluant les règnes animal et végétal, a permis de faire le point sur les conséquences écologiques, pathologiques et génétiques de ces pratiques.

Les conséquences génétiques ont la particularité de n'apparaître que lorsqu'il y a croisement entre taxon autochtone et taxon introduit. Ces croisements n'auront d'impact génétique à long terme sur une population naturelle que s'ils induisent un effet héréditaire. Ainsi, une hybridation dont les descendants $F 1$ sont stériles n'aura pas de conséquence génétique ultérieure : la juxtaposition de gènes issus de taxons différents, telle qu'on la rencontre dans le génome des $\mathrm{F} 1$, ne sera pas transmise aux générations suivantes. Dans ce cas, la modification génétique ne concerne donc qu'une génération d'organismes stériles. D'un point de vue pratique cependant, il faut se méfier d'un diagnostic trop rapide de stérilité (basé sur l'analyse morphologique de peu d'individus par exemple). Dans le cas de I'hybridation naturelle entre Alburnus alburnus et Rutilus rubilio dans le lac Prespa (Grèce), la quasi-stérilité des $\mathrm{F} 1$ a cependant permis une introgression d'un nombre extrêmement faible de gènes d'une espèce dans l'autre, estimée approximativement à 1 sur 5000 (BERREBI et al., 1989). A l'échelle de temps humaine, les conséquences génétiques sont négligeables, mais il est possible qu'à l'échelle géologique, les deux espèces voient leur évolution influencée par ce flux génique.

L'absence de conséquence génétique héréditaire suite à une introduction ne signifie pas une absence totale de conséquence. L'existence de ces F1 stériles peut modifier l'équilibre écologique. Cependant, ce type de perturbations n'entre pas dans les objectifs de ce texte.

Si l'hybridation consécutive à une introduction produit des F1 fertiles (ce qui aboutit à la production d'hybrides " post-F1 ", essentiellement des backcrosses), il y a introgression, cela signifie que la population native qui subit cet apport est modifiée génétiquement. Cette modification affecte l'intégrité des taxons parentaux et peut modifier leurs caractéristiques biologiques.

La substitution de gènes d'une espèce par des gènes d'une autre est rarement anodine. Les relations épistatiques (entre gènes différents) sont très importantes dans le fonctionnement des cellules et par là des organismes, des populations et des taxons. Les allèles caractérisant les gènes d'un organisme naturel équilibré ont été sélectionnés lors d'un long processus évolutif qui a abouti à la combinaison la meilleure possible, compte tenu des contraintes physiologiques, de l'histoire évolutive de l'organisme, et des caractéristiques du milieu. Si ces dernières se sont modifiées (évolution climatique par exemple), les combinaisons génétiques des organismes s'y sont adaptées en se modifiant, par le jeu des mutations et de la sélection, en puisant dans le polymorphisme génétique populationnel. 
Cette combinaison, issue d'un équilibre sans cesse modifié par le milieu, les migrations, les mutations, est appelée " complexe de gènes coadaptés ". L'introgression peut donc provoquer la rupture de complexes de gènes coadaptés. La conséquence de ces ruptures est aléatoire. Même si on a décrit des cas d'amélioration des caractéristiques des organismes modifiés (effet hétérosis, par exemple chez le maïs (SCHULER, 1954) ou chez Spartina anglica (MARCHANT, 1967)), la grande majorité des cas connus aboutit à une modification neutre ou néfaste (" outbreeding depression ", TEMPLETON, 1986). POTEAUX (1995), dans l'Orb, a montré que la valeur adaptative des truites croisées (fario domestique introduite $x$ fario méditerranéenne sauvage) était probablement plus faible que celle des truites sauvages, une certaine incompatibilité génomique ayant été décelée malgré la proximité phylogénétique des deux taxons (croisements infraspécifiques), bien que GUYOMARD (1997) estime que cette incompatibilité ne peut être forte. Un cas similaire d'hybridation intraspécifique a été décrit chez les saumons américains (WOOD et FOOTE, 1990). La rupture de complexes de gènes coadaptés provoque un mauvais fonctionnement de l'organisme dû à de multiples causes (chaque fois qu'un gène du taxon A fonctionne moins bien dans un environnement composé de gènes du taxon $B$ ). II en résulte une baisse intrinsèque de valeur adaptative.

La cause principale d'une baisse de valeur adaptative est parfois connue : PHILIPP (1991) a montré, dans le cas de l'introduction du "Florida largemouth bass " (Micropterus salmoides floridanus) dans l'aire du "Northern largemouth bass" (M. s. salmoides), que la résistance au froid de ce dernier était abaissée suite à l'introgression. Dans ce cas précis, bien que des ruptures de complexes de gènes coadaptés aient probablement eu lieu, l'effet principal de l'introduction est plutôt une " rupture d'adaptation au milieu ", ici la résistance au froid. II s'agit là d'un second type de conséquences prévisibles : l'adaptation d'une population à son milieu est due à de subtiles sélections " dirigées " par l'environnement. Ces micro-adaptations locales, issues de milliers d'années de sélection, peuvent être mises à mal par l'introduction de taxons qui sont adaptés à d'autres milieux, ou pire, qui sont adaptés à des conditions artificielles quand les individus introduits proviennent de souches domestiquées qui ont subi la sélection, sévère cette fois, qu'est la domestication.

L'objectif de ce texte est de mettre en relief les conséquences de certaines introductions. Parce qu'un mécanisme essentiel des organismes vivants, leur " machinerie " génétique, est perturbé, les risques pour l'avenir sont importants, allant jusqu'à l'extinction.

A partir de nombreux cas particuliers, les relations entre taxons introduits et taxons natifs vont être classées selon des critères génétiques. Les cas français connus sont peu nombreux, aussi des exemples étrangers pertinents seront cités. Cette classification envisage tous les cas depuis l'incompatibilité totale jusqu'à l'inter-fécondité totale. Il est à noter que cet éventail très large ne se traduit par un impact génétique que pour les types 3 et 4 où la descendance est fertile.

Ce classement servira de support à une discussion générale sur les conséquences concrètes de ces "introductions introgressives", sur les possibilités de revenir sur leurs conséquences néfastes et sur ce qu'il convient de faire de façon préventive pour les éviter. II est généralement reconnu que l'hybridation est un des risques majeurs et immédiats des introductions (CRIVELLI et MAITLAND, 1995).

\section{ÉCHELLE DES CONSÉQUENCES GÉNÉTIQUES}

Les différents degrés de conséquences proposés ci-dessous vont de l'effet nul (type 0) à l'hybridation sans limite (type 4) pouvant aboutir à la formation d'un nouveau taxon intermédiaire homogène et panmictique. Bien que ce ne soit pas toujours aussi simple, ces types forment une suite continue de situations, depuis l'hybridation entre taxons fortement différenciés (espèces), généralement dans les types 0 à 2 , jusqu'à l'hybridation entre taxons très proches (niveau infraspécifique), surtout dans le type 4. 


\section{Type 0 : aucun effet}

Ce sont les espèces pour lesquelles l'interfécondation est impossible malgré la mise en contact des produits génitaux. Ce contact, même s'il est sans conséquence, sous-entend une certaine promiscuité et une coïncidence de période de reproduction. Bien sûr, entre espèces qui n'ont aucun contact (vivant dans des milieux séparés) la question ne se pose pas. Ainsi, la plupart des espèces à fécondation externe (lamellibranches, poissons), ou dont un type de gamètes est libéré dans le milieu (plantes supérieures), et utilisant l'eau comme vecteur, sont susceptibles d'entrer en contact fortuit. Dans la grande majorité des cas, l'incompatibilité est totale pour des raisons évidentes de non-concordances morphologique et physiologique des gamètes.

\section{Type 1 : hybrides non viables, mais effets possibles}

Bien que proches du cas précédent, les conséquences de ce type d'interactions sont nettement distinctes : il y a au moins un début de segmentation embryonnaire qui peut aller au plus à la naissance d'hybrides à vie très courte. On sait par exemple que le sperme de carpe déclenche expérimentalement la segmentation de l'oeuf du barbeau commun sans jamais donner d'hybride viable. Ce phénomène est d'ailleurs exploité pour produire une descendance gynogénétique expérimentale chez le barbeau (CASTELLI, 1994). Dans la nature, et spécialement lors de l'introduction d'un nouveau taxon, la difficulté de l'observation de ce type de segmentation embryonnaire explique le peu de cas signalés. Les conséquences peuvent être une certaine mortalité des oeufs qui ne seraient pas fécondés ou, chez les mammifères, des avortements gênant la reproduction normale de l'espèce indigène.

Un des rares exemples d'interactions de type 1 est peut-être celui du vison d'Europe (Mustela lutreola) concurrencé par le vison d'Amérique ( $M$. vison) initialement élevé en captivité pour sa fourrure. MAURIN (1997) signale que l'espèce américaine est en pleine expansion en France et semble pouvoir remplacer l'espece locale. Alors que MAIZERET et al. (1995) signalent que les deux espèces sont difficiles à distinguer morphologiquement (distinction basée sur la présence d'une tache blanche plus large chez l'espèce autochtone, et sur la taille), ARTOIS et al. (1995) précisent une nette différenciation immunologique et prédisent une hybridation possible mais à descendants stériles. En fait, peu d'observations sont disponibles sur ces interactions, mais on soupçonne la possibilité de copulations interspécifiques donnant lieu à des avortements qui participeraient (dans quelle mesure ?) au déclin de l'espèce européenne.

\section{Type 2 : production de $\mathbf{F} 1$ stériles}

Ce type d'hybridation, bien qu'ayant des conséquences essentiellement écologiques, est à surveiller de près. II est en effet souvent difficile d'affirmer que les F1 sont totalement stériles (BERREBI et al., 1989). De plus, MINA (1991) signale comme conséquence potentiellement dramatique de la production de F1 stériles, ce qu'il nomme la " reproductive sex-extermination ". Selon lui et selon ZASLAVSKY (1967, in MINA, 1991), si on introduit en grand nombre un taxon capable de former des F1 stériles avec le taxon résident, la production en descendants de ce dernier sera réduite d'autant. Si le taxon introduit est en plus grande densité (on parle de contact dispersif), le taxon résident ira à l'extermination, car il se reproduira surtout par hybridation, pour des raisons de probabilité de rencontre, et ses gènes seront perdus dans l'impasse des F1. Cet auteur ne donne pas d'exemple concret, mais on exploite ce principe en lutte biologique avec des mâles stériles.

Le cas de l'hybridation entre les graminées côtières et estuariennes, Spartina maritima autochtone et $S$. alterniflora, sans doute introduite des Amériques au siècle dernier (GUENEGOU et al., 1988) est particulièrement instructif, bien qu'il se situe aux limites du domaine des eaux douces. Les hybrides produits à la suite de cette introduction ont donné plusieurs formes, qui ont été classées dans le " complexe 2 " (MOBBERLEY, 1956) ou " complexe $S$. townsendii " comprenant une forme stérile diploïde, $S$. townsendii qui est un F1, divers polyhaploïdes stériles et une forme fertile tétraploïde, détaillée dans le "type 3 " (MARCHANT, $1967 ; 1968$ ). 


\section{Type 3 : production de F1 fertiles, introgression limitée}

L'hybridation entre taxon autochtone et taxon introduit peut ne donner qu'occasionnellement des descendants fertiles (donc de rares stades " post- $F 1$ "). II est tentant de considérer ce cas comme sans importance. Cependant, nous atteignons là un niveau d'interactions le plus souvent irréversible. La faible intensité du flux génique entre les deux taxons possède en effet la potentialité de modifier à long terme le taxon natif. Ce flux peut devenir intense à l'occasion d'une modification d'un paramètre écologique comme le climat. La surveillance de ce type d'hybridation s'impose.

- Lors de l'hybridation entre Spartina maritima autochtone et S. alterniflora introduite, la forme hybride nommée $S$. anglica, apparue il y a seulement un siècle en Grande-Bretagne, a montré une telle capacité de colonisation que son aire de répartition ne cesse de s'étendre en France le long des côtes armoricaines, mais aussi dans le monde entier. II faut noter cependant que l'origine hybride de $S$. anglica n'a pas été prouvée expérimentalement, mais cette hypothèse est généralement acceptée (MARCHANT, 1968). Alors que la forme hybride diploïde semble relativement "sage ", ne serait-ce que du fait de sa stérilité, la forme tétraploïde, issue d'un phénomène d'allopolyploïdie (additionnant les chromosomes des espèces parentales), a acquis non seulement la fertilité, mais un dynamisme exceptionnel. Sur le littoral de la Manche, on craint que cette forme supplante la forme naturelle $S$. maritima. L'hybride a acquis une autonomie reproductive, probablement due au "saut de ploïdie ", et on ne sait pas si des backcrosses sont possibles (GRAY, 1986) : ce dont on est sûr, c'est de l'interfertilite des individus tétraploïdes entre sux. Dans la mesure où on attribue l'apparition de la forme tétraploïde à un événement unique survenu en Grande-Bretagne, l'introgression entre les taxons parentaux est nécessairement faible, ce qui classe ce cas dans le type 3 (introgression limitée). Cette introgression a cependant donné lieu à une forte invasion : Europe, Australie, Chine, Amérique (GUENEGOU et LEVASSEUR, 1989).

- La truite fario est une des espèces de poisson les plus manipulées et commercialisées à travers le monde. Elle s'hybride avec le saumon atlantique en divers endroits du monde où la truite a été introduite (VERSPOOR et HAMMAR, 1991). Dans ces régions, malgré une forte sympatrie des aires de ponte, la proportion d'hybrides ne dépasse pas quelques pourcents. En fait, une des limitations à l'introgression vient du fait que seul le croisement " truite femelle $x$ saumon mâle "semble possible à Terre-Neuve (MACGOWAN et DAVIDSON, 1992), tandis qu'assez bizarrement, c'est le croisement inverse qui serait seul possible en Espagne (BEALL et al., 1997). On a montré expérimentalement que la production de $\mathrm{F} 2$ et de backcrosses était possible (NYGREN et al., 1975). Cependant, ces backcrosses ont été rarement analysés au niveau de leurs gènes. JOHNSON et WRIGHT (1986) ont montré qu'il pouvait s'agir de gynogenèses et de production de triploïdes. La rareté du phénomène dans la nature et le peu de connaissances génétiques que nous en avons rendent provisoire le classement de ce cas dans le type 3.

- Salvelinus fontinalis (saumon de fontaine), espèce nord-américaine introduite dans des cours d'eau où elle était absente, produit surtout des $\mathrm{F} 1$ avec $\mathrm{S}$. confluentus (espèce native : bull trout) : sur un échantillon de 53 hybrides analysés, 50 étaient des F1 et 3 seulement possédaient des génotypes recombinés (LEARY et al., 1995).

- Le canard colvert, Anas platyrhynchos, est très employé pour agrémenter les plans d'eau et a ainsi été introduit dans plusieurs pays. II s'est croisé avec $A$. poecilorhynca en Nouvelle-Zélande et Australie, avec $A$. ribripes à l'est des États-Unis et avec $A$. diazi au sudouest de ce pays. Cette forte compatibilité entre espèces du même genre semble cependant s'accompagner d'une moins bonne valeur adaptative des hybrides, dont le taux dépasse rarement $10 \%$ de présence (CADE, 1983 ; MACDONALD et al., 1989).

\section{Type 4 : interpénétration des taxons, homogénéisation possible à terme}

Ce dernier type se situe le plus souvent (mais pas toujours) à un niveau de différenciation infraspécifique. II concerne des espèces présentant des sous-unités faiblement 
différenciées (sous-espèces ou nettement en dessous) susceptibles d'entrer en contact secondaire. Le risque d'aboutir à un nouveau taxon à partir d'un mélange plus ou moins équilibré des espèces parentales est grand. Plusieurs cas d'espèces considérées comme issues d'hybridation sont connus, mais ils ne sont pas le fruit d'introductions mais de contacts naturels. C'est le cas de barbeaux espagnols (MACHORDOM et al., 1990), d'une sous-espèce de Cobitis taenia en Italie (BIANCO, 1995) ou d'un cyprinidé américain du genre Rhinichthys (GOODFELLOW et al., 1984).

- Le cas des truites fario domestiques déversées en région méditerranéenne est largement détaillé dans ce fascicule (POTEAUX et BERREBI, 1997). La forme domestique, déversée en grande quantité, est en fait constituée d'un ensemble de souches domestiques dont les origines remontent au début du siècle et dont les premiers géniteurs sont probablement scandinaves, de type atlantique. Les populations réceptrices sont ici de type méditerranéen. Ces repeuplements, justifiés par la surpêche, sont quelquefois fortement introgressifs en région méditerranéenne (BARBAT-LETERRIER et al., 1989; LARGIADĖR et SCHOLL, 1995), et ne semblent que très peu limités par les incompatibilités génomiques qui ont été décelées (POTEAUX, 1995). Dans ce cas, l'introduction d'un taxon étranger se fait donc par l'intermédiaire d'un stade de domestication complexe, dont on peut se demander ce qu'il a gardé de ses caractéristiques sauvages d'origine.

- Le cas de la carpe en Camargue est unique. II s'agit d'une forme introduite très récemment (la carpe cuir, qui est une forme domestique ayant perdu ses écailles) qui semble s'hybrider avec une forme introduite à l'époque romaine, la carpe commune, considérée comme parfaitement acclimatée depuis de multiples générations. La forme hybride, morphologiquement reconnaissable, devient prépondérante et va probablement faire disparaitre la forme camarguaise ancienne. Une situation comparable a été observée en Australie (ROSECCHI et al., 1997).

- L'ombre commun (Thymallus thymallus) est une des espèces ayant le plus souffert des barrages successifs qui ont morcelé son habitat initial dans le Haut-Rhône, entre Genève et Lyon. En compensation aux aménagements, des alevinages sont effectués chaque année. Les poissons déversés sont issus d'une souche élevée à la pisciculture du CSP d'Augerolles (Puy de Dôme) depuis 1973. Cette souche a été créée à partir d'oeufs importés de Bavière et de Finlande. Cette origine mixte confère à cette souche des caractéristiques génétiques particulières (PERSAT et EPPE, 1997). Certains allèles caractéristiques des différentes origines ont permis de conclure que très peu d'ombres du Rhône semblent être des produits d'Augerolles, que l'impact important des repeuplements ne peut pas découler des opérations de ces dernières années mais plutôt des premiers alevinages consécutifs à la grave pollution de 1978 (vidange du barrage de Génissat). Là où l'ombre sauvage a survécu, l'introgression s'est avérée limitée. Si, en définitive, il s'agit bien d'introduction de taxons étrangers (Bavière, Finlande), comme dans le cas de la truite fario, on ne peut pas affirmer qu'il s'agisse de taxons physiologiquement différents. Toutefois, la résistance des populations sauvages restées en bonne condition montre bien qu'on ne peut pas assimiler les formes de repeuplement à des formes sauvages. II n'est cependant pas possible de dire si cette faible pénétration des ombres de pisciculture est due à leur origine étrangère ou bien seulement à leur domestication.

- La truite marbrée (Salmo trutta marmoratus ou Salmo marmoratus selon les auteurs) est endémique des bassins adriatiques d'Italie, Slovénie, Croatie, Bosnie-Herzégovine, Monténégro et Albanie (POVZ, 1989). La truite fario (Salmo trutta fario), principale souche de repeuplement en Europe, a été introduite à l'intérieur de l'aire de répartition de la truite marbrée. Si, en Italie, ces deux formes semblent ètre naturellement présentes dans des parties distinctes des mêmes cours d'eau, les introductions généralisées de fario y ont fait souvent disparaître, par hybridation, des populations purement marmoratus (BIANCO, 1995; GIUFFRA et al., 1996). L'introduction introgressive a également été montrée dans le bassin du Pô, en Suisse italienne (LARGIADĖR et SCHOLL, 1995). En Slovénie, la truite marbrée est également fortement hybridée par la truite fario naturellement absente du bassin adriatique 
(OCVIRK, 1989 ; BUDIHNA et OCVIRK, 1990). Cette introduction date de 1906 (POVZ, 1995). Des études allozymiques récentes ont montré que ces introductions ont été constituées aussi bien de fario de pisciculture que de fario du Danube (BERREBI, 1995a). Un programme de réhabilitation de la truite marbrée est en cours dans ce pays (JESENSEK, 1994 ; POVZ et al., 1996).

- L'érismature rousse (Oxyura jamaicensis) est un anatidé américain introduit à des fins de loisir en Grande-Bretagne vers 1950. L'effectif de l'espèce n'a cessé d'augmenter depuis. L'érismature rousse s'est étendue au continent, et la France en héberge depuis les années 70 (HUGHES et GRUSSU, 1994). L'espèce introduite s'hybride facilement avec l'espèce autochtone $O$. leucocephala (l'érismature à tête blanche) dont les dernières populations européennes vivent en Espagne, donnant des hybrides fertiles (URDIALES et PREIRA, 1993). Cette hybridation a été confirmée à la fois par des données expérimentales et par des observations dans la nature. Bien que le processus n'en soit qu'à ses débuts, les auteurs s'accordent à dire que l'érismature rousse importée gagne du terrain sur l'espèce européenne (HUGHES, in press) et que son extension, voire la disparition de l'érismature blanche, n'est qu'une question de temps. En outre, cette hybridation s'oppose au projet de réintroduction de l'érismature à tête blanche en France, d'où elle a disparu dans les années 70 (PERENNOU, 1997).

- ALLENDORF et al. (1980), LEARY et al. (1984), GYLLENSTEN et al. (1985) ou FORBES et ALLENDORF (1991) ont décrit les conséquences catastrophiques de l'introduction de la truite arc-en-ciel (O. mykiss, "rainbow trout ") dans des peuplements natifs de "cutthroat trout " (O. clarki). Dans le nord-ouest des États-Unis, l'introgression a provoqué la formation de populations composées uniquement d'hybrides (" hybrid swarms "), où les gènes parentaux des deux espèces sont répartis au hasard chez tous les individus. Cette situation est considérée comme une extinction locale de $O$. clarki.

- Cas similaire : aux États-Unis, le "Guadalupe bass" (Micropterus trecull) est une espèce autochtone qui a subi l'introduction du " smallmouth bass" $(M$. dolomieu), avec lequel il semble s'hybrider sans limite (WHITMORE, 1983 ; MORIZOT et al., 1991).

- Même situation signalée dans le Montana (U.S.A.), où une sous-espèce locale de la truite arc-en-ciel, O. mykiss lewisi a subi le même type d'extinction génétique (LEARY et al., 1984 ; ALLENDORF et LEARY, 1988), et n'existe plus à l'état pur que dans $2,5 \%$ de son aire initiale (LIKNES et GRAHAM, 1988).

- Dernier cas de la série, pour lequel $60 \%$ de la répartition naturelle du "Pecos pupfish" (Cyprinodon pecosensis) a subi l'invasion introgressive du "sheepshead minnow" (C. variegatus) après introduction (WILDE et ECHELLE, 1992).

- Les saumons Sockeye et Chum ont vu leur densité décliner dans le fleuve Columbia (U.S.A.) à cause, entre autres, d'un changement de comportement (homing) après repeuplement (LEE, 1993 in FONTENELLE, 1994). Le déclin du saumon coho au nord de la Californie (BROWN et al., 1994 ; FONTENELLE, 1994) semble être également en partie lié aux pratiques de repeuplement.

- Dans le cas des grenouilles vertes du complexe esculenta, le risque d'introgression est jugé important (voir les détails dans NEVEU, 1997) du fait des importations d'individus vivants d'origines très variées, pour la consommation (DUBOIS, 1983) ou comme animaux de laboratoire (NEVEU, 1989). Cependant, les données sont incomplètes (CROCHET et al., 1995) et ne permettent pas de confirmer l'existence d'hybridations entre sous-espèces autochtones (complexe esculenta au nord-est de la France; complexe perezi au sud-ouest) et entre sous-espèces autochtones et introduites; et encore moins d'estimer l'importance du phénomène. 


\section{DISCUSSION}

Nous avons vu que les exemples qui mettent en évidence les influences palpables de certaines introductions sont très nombreux. Quels enseignements peut-on en tirer?

\section{Quels sont les risques au niveau du taxon?}

BUSACK et CURRENS (1995), et NELSON et SOULÉ (1987), détaillent les risques de modification génétique tels que perte de variabilité génétique intrapopulationnelle par dérive ou consanguinité, ou perte de variabilité interpopulationnelle qui correspond à une baisse de biodiversité au niveau de l'espèce. II peut paraître surprenant de dire que l'introduction d'un taxon différent aboutit à une perte de biodiversité alors que, dans un premier temps, il y a enrichissement par des allèles exotiques. Tant que l'introgression est modérée, et même jusqu'à $50 \%$, il y aura gain de variabilité. Au delà, il y a risque de perte. NELSON et SOULÉ (1987) proposent un cas hypothétique où le mélange à part égale de deux taxons compatibles ne provoque aucune perte génique dans la population résidente. Mais, quand la population hybridée arrive à l'équilibre, la variance génotypique en général et certains génotypes homozygotes du taxon résident en particulier, sont réduits de moitié. Au niveau régional, et de façon plus intuitive et globale, si une espèce occupe plusieurs bassins hydrographiques, il est probable qu'une certaine différenciation existe entre ses populations, que ce soit par dérive génétique ou par micro-adaptation aux hétérogénéités écologiques. Le fait de déverser un taxon domestique homogène en grande quantité sur toute l'aire tendra bien sûr à réduire les différences génétiques entre populations introgressées.

\section{Quels sont les risques au niveau de la population?}

II est possible que la contre-sélection des gènes étrangers fasse que seuls les allèles neutres (ne provoquant aucun changement dans le fonctionnement de l'organisme) soient conservés. Les analyses génétiques détecteront malgré tout l'introgression neutre.

A l'opposé, si l'introgression concerne des gènes adaptatifs, plusieurs phénomènes peuvent apparaitre (LEARY et al., 1995 ; BUSACK et CURRENS, 1995) :

- rupture de complexes de gènes, ce qui entraîne une baisse générale de valeur adaptative, avec tout un cortège de mauvais fonctionnements dont le moindre connu est l'augmentation de l'asymétrie fluctuante;

- rupture des adaptations locales, conséquence d'une baisse de biodiversité globale chez une espèce. C'est le cas en particulier quand une souche de pisciculture supplante progressivement toutes les variétés locales d'une espèce, ces organismes ont alors perdu les micro-adaptations aux particularités de chaque cours d'eau habité, ce qui baisse également la valeur adaptative locale.

\section{L'introgression est-elle réversible?}

Eradiquer les représentants d'une introduction dont les conséquences sont reconnues néfastes peut être tentant. Mais quand l'introduction a provoqué une introgression, cette éradication est plus que problématique. Bien que les individus introduits qui participent à la reproduction soient souvent désavantagés par la domestication, qui les a rendus inaptes aux conditions naturelles (en grande partie par déficit d'apprentissage), quand il y a introgression, une partie, même faible, de ces individus introduits a réussi à se reproduire avec les individus sauvages (moins de 0,5\% chaque année dans le cas de la truite fario dans le sud de la France, BEAUDOU, 1993). Les descendants F1 fertiles produisent généralement des backcrosses de première génération avec une des espèces parentales, la production de F2 (croisement entre deux F1) est improbable dans le milieu naturel, dominé par les individus sauvages. Ces backcrosses sont au début composés de $3 / 4$ de gènes sauvages et $1 / 4$ de gènes introduits. Leur succès reproducteur est alors bien meilleur, pour deux raisons potentielles : (i) si le facteur " inadaptation " dû à l'élevage en pisciculture est prépondérant, la naissance dans la nature des backcrosses élimine ce handicap; (ii) si les incompatibilités génomiques ont un rôle dans cet 
échec, un assemblage génomique $3 / 4-1 / 4$ sera plus équilibré qu'un assemblage $1 / 2-1 / 2$. Ces stades constituent alors le départ d'une dissémination irrésistible des gènes introduits, même si elle est en partie contre-sélectionnée (POTEAUX et al., soumis). Dans un premier temps, c'est au niveau de la méiose que les chromosomes des deux taxons vont se mélanger pour former des combinaisons génétiques nouvelles. Puis, par le jeu des crossing-over, chaque chromosome sera constitué d'une mosaïque plus ou moins fine. Or, même si le pourcentage de gènes introduits est faible, à terme, chaque individu sera introgressé au hasard dans son génome de ce même pourcentage, par des gènes de la forme introduite. Bien sûr, cette description stochastique du phénomène n'est pas parfaitement exacte : divers facteurs tels que sélection, incompatibilité, pseudo-linkage, vont perturber cette recombinaison, le plus souvent en la limitant. Mais même ralentie et irrégulière, cette installation des gènes étrangers aura lieu.

LEARY et al. (1995) parlent d'éradication et de restauration génétique. Dans le cas des poissons, ils proposent la capture des spécimens introduits ou introgressés, ou l'utilisation de poison, même si de leur propre aveu, l'utilisation de ce dernier " peut causer certains problèmes "! MINCKLEY (1995) cite quelques cas d'éradication réussie dans de petits cours d'eau.

Quand l'éradication n'est pas possible, LEARY et al. (1995) proposent la restauration génétique, par l'emploi de souches locales lors d'introductions ultérieures. Ils signalent que même si la souche locale servant à la réhabilitation a un bon succès, les dépenses en temps et en argent seront très importantes. Dans le meilleur des cas, on obtiendra la réduction de moitié de l'introgression à chaque génération, si on déverse autant d'individus de la souche autochtone qu'il y a d'individus en place, et si tous se reproduisent! Les auteurs pensent qu'une opération de restauration peut être efficace si l'introgression est inférieure à $10 \%$. Au delà, seule l'éradication aura un effet. MINCKLEY (1995) détaille les conditions dans lesquelles une restauration à partir de formes sauvages est possible.

Un cas européen peut être cité : la restauration de la truite marbrée en Slovénie. Une étude génétique a été menée en 1993 et 1994 dans le bassin de la Soca (nommée Isonzo en Italie), domaine naturel de Salmo trutta marmoratus. Un vaste programme de restauration est en cours (JESENSEK, 1994 ; POVZ et al., 1996). Des alevins de truite marbrée sont produits en grand nombre à partir de géniteurs sauvages dont la pureté a été contrôlée par analyses allozymiques (BERREBI, 1995a). Un stage prolongé en cours d'eau "pépinière " assure une bonne survie des truitelles d'un an, une fois libérées dans les rivières où a lieu l'hybridation. Nous ignorons si cette restauration par "dilution des hybrides " est efficace et un programme de contrôle est en cours d'application (analyses génétiques et marquage).

Un cas comparable d'utilisation de souche locale a donné des résultats négatifs : celui du lac Tom Tom dans le Montana (U.S.A.). Ce lac contenait des "Yellowstone cutthroat trout " d'origine domestique et son émissaire contenait encore des "Westslope cutthroat trout" naturelles quasiment pures. Pour favoriser la "Westslope" naturelle, des truitelles de cette souche locale ont été produites et déversées dans le lac durant plusieurs années. L'effet de cette restauration a été négatif : des "Yellowstone " du lac ont émigré dans l'émissaire, augmentant ainsi l'introgression dans le cours d'eau, où la forme sauvage s'était maintenue pure jusque-là (LEARY et al., 1984).

\section{Introgression et extinction}

Selon LEARY et al. (1995), l'introgression entre taxons équivaut à l'extinction, dans une perspective génétique du moins, de la population réceptrice : les génotypes natifs (sensu multilocus) ont disparu dès lors que l'introgression s'est généralisée à un bassin et que l'importance de cette introgression (quelques pourcents) implique que tout individu de la population a été modifié par le jeu des recombinaisons génétiques. Cette extinction signifie que les génotypes natifs ont été mêlés de façon irréversible avec des génotypes d'un taxon différenciè. Dans certains cas, ces hybridations peuvent donner naissance à une entité nouvelle, qui pourrait prendre statut d'espèce dans l'avenir. Bien sûr, les pratiques d'introduction sont dans leur grande majorité trop récentes pour donner lieu à de tels bouleversements. 
Cependant, plusieurs exemples, dont on ne sait s'ils sont naturels ou dûs à l'homme, sont connus, comme celui de Cobitis taenia zanandreai en Italie (BIANCO, 1995). Dans d'autres cas, une vraie extinction physique (baisse de densité jusqu'à disparition) est redoutée localement comme pour l'érismature à tête blanche en Espagne (PERENNOU, 1997) ou les saumons du nord-ouest des États-Unis. En effet, les saumons coho (Oncorhynchus kisutch), chinook (O. tshawytscha) et chum (O. keta) ont vu leur densité baisser, en partie à cause des introductions de saumons d'élevage (WRIGHT, 1993 ; JOHNSON et al., 1991), et spécialement dans la rivière Columbia (NEHLSEN et al., 1991). II semblerait que la proportion grandissante de saumons d'origine domestique perturbe fortement leurs caractères naturels issus de l'adaptation au milieu (FONTENELLE, 1994). D'une part, les formes sauvages sont littéralement diluées par les formes domestiques et, d'autre part, l'action humaine (repeuplement, aménagement du milieu) s'exerce de façon inégale selon que l'espèce est d'un grand intérêt économique (chinook, coho) ou peu prisée (FONTENELLE, comm. pers.).

Selon HUBBS (1955), les introductions d'espèces exotiques induiraient un risque plus important d'hybridation avec les espèces autochtones qu'entre deux espèces naturellement sympatriques. En allopatrie, les barrières reproductrices ne seraient pas forcément érigées, simplement parce que ce processus n'est pas indispensable à la spéciation, comme c'est le cas en sympatrie. Cette hypothèse est particulièrement bien illustrée par le cas de l'hybridation entre truite fario et saumon atlantique : dans l'aire de répartition commune des deux espèces (Grande-Bretagne), à latitude sensiblement égale $\left(50^{\circ} \mathrm{N}\right)$, la proportion maximale d'hybrides naturels dans les populations est inférieure à 5 pour mille, tandis qu'en zone d'introduction (Terre-Neuve), cette proportion est doublée, voire décuplée (MACGOWAN et DAVIDSON, 1992). HUBBS (1955) suggère également qu'une forte disparité de densité des deux espèces susceptibles de se croiser favoriserait l'hybridation. C'est particulièrement le cas lors des introductions.

\section{RECOMMANDATIONS}

\section{Le taxon, notion à prendre en compte}

L'espèce, telle qu'elle est définie par son isolement reproducteur vis-à-vis des autres espèces, n'est pas le seul niveau d'intégration qu'il faut considérer. De nombreuses espèces sont composées de "sous-espèces " interfertiles et même de "groupes " distincts par de nombreux caractères malgré une interfertilité presque totale. Le cas de la truite fario est exemplaire. Il est à présent un des modèles animaux les plus analysés par marqueurs biochimiques et offrant une grande gamme de cas. Quand des truites de souche domestique de type atlantique sont déversées dans les régions méditerranéennes françaises (POTEAUX et BERREBI, 1997) la compatibilité est partielle mais bonne. Si la contre-sélection des formes hybrides ou des allèles domestiques est certaine, elle semble cependant incapable de "purifier " une population introgressée, rendant donc l'introgression irréversible.

Les introgressions infraspécifiques sont peu connues, simplement par ignorance de la phylogénie fine de l'espèce considérée. C'était le cas en France où pendant longtemps, on ne se doutait pas qu'on effectuait des mélanges contre nature en repeuplant nos rivières en truites d'élevage.

La première des recommandations est donc bien d'augmenter les connaissances génétiques sur les espèces qui peuplent nos cours d'eau, par des techniques d'une efficacité maximale dans la détection de taxons infraspécifiques (marqueurs moléculaires). Cette connaissance semble un préalable indispensable, un " point zéro " qui manque cruellement dans de nombreux cas d'introduction.

\section{Le principe de précaution : l'échelle du bassin hydrographique}

L'observation des quelques cas français et des nombreux cas étrangers exposés dans ce texte montre que l'introgression génétique est un phénomène redoutable parce qu'il semble 
le plus souvent irréversible. Même si les gestionnaires nord-américains ont tenté des restaurations ou des éradications, même si les gestionnaires slovènes établissent, aidés par un bénévolat traditionnel développé, un programme de restauration de la truite marbrée, force est de constater que l'objectif est ambitieux et que rien ne garantit son succès. Seule une inversion de la courbe d'introgression (réduction du taux d'introgression) est envisageable. Plus le bassin considéré est petit, plus l'homme pourra influer sur le taux d'introgression. Cependant, le retour à la pureté génétique du taxon reste problématique.

Alors, devant ce risque d'introgression définitive pouvant provoquer la perte d'une forme de vie originale, le mieux est d'agir préventivement et d'éviter toute action de type " apprenti sorcier".

L'évolution des espèces fortement inféodées aux cours d'eau est particulière : l'isolement entre populations d'une même espèce est plus important que dans le milieu terrestre. De ce fait, il est normal que des différences se creusent avec le temps entre ces populations. La difficulté est de choisir le niveau quantitatif de différenciation à partir duquel une protection est souhaitable. En d'autres mots, dans le domaine des repeuplements de poissons, si on conçoit bien que des souches scandinaves ne doivent pas être introduites dans le Rhône, que penser de l'introduction dans le Rhône de souches issues de la Seine, de la Garonne, ou de l'Hérault tout proche? Tout cours d'eau contient-il, pour une espèce donnée, une forme digne d'être protégée?

Les études génétiques ont souvent montré des différenciations inter cours d'eau, qui étaient difficiles à détecter par la morphologie. Dans le cas de la truite, celles qui habitent naturellement les cours d'eau français des Pyrénées Orientales se jetant en Méditerranée appartiennent à la forme méditerranéenne de la sous-espèce fario (BARBAT-LETERRIER et al., 1989 ; BERREBI, 1995b). Celles de l'Ariège par exemple, dont certains affluents ne sont parfois qu'à quelques kilomètres d'un affluent méditerranéen, sont de type atlantique. Des études ont montré les perturbations causées par le rempoissonnement de rivières méditerranéennes par des souches atlantiques de pisciculture (POTEAUX et BERREBI, 1997). Du point de vue de la biodiversité, il s'agit là d'introductions perturbatrices qu'il faut proscrire. Par contre, l'étude allozymique des différents bassins méditerranéens français a montré une remarquable homogénéité des formes sauvages. Ces connaissances récentes nous permettent de recommander de limiter les manipulations à des introductions de truites d'un cours d'eau méditerranéen à un autre cours d'eau méditerranéen. Encore faudra-t-il tenir compte de nouveaux marqueurs moléculaires (microsatellites) qui montreront, lorsque les études seront publiées, sans doute des subdivisions nouvelles (POTEAUX, 1995). Les introductions de truites d'un cours d'eau atlantique à un autre cours d'eau atlantique ne peuvent cependant pas encore être approuvées puisque la structuration génétique des peuplements atlantiques français de cette espèce n'est pas encore bien connue et que de fortes hétérogénéités nord-sud existent (BERREBI et BEAUDOU, 1991 ; données non publiées).

Dans la majorité des cas, la description génétique de la biodiversité des taxons d'eau douce n'est pas disponible : comment se comporter vis-à-vis de toute introduction risquant de provoquer une introgression?

D'un point de vue génétique, le principe de précaution nous dicte de ne pas mettre en présence des formes qui n'auraient aucune chance de se rencontrer sans l'action de l'homme : celles qui n'habitent pas le même bassin hydrographique. Cela s'appliquerait le plus souvent à un niveau infraspécifique, dans le but de préserver la biodiversité (risque majeur) et pour éviter des incompatibilités ou des inadaptations locales abaissant la valeur adaptative des formes hybrides (risque secondaire). Si on accepte ce principe de précaution " par bassin ", la transplantation d'un taxon aquatique d'un bassin à l'autre n'est acceptable qu'après analyse génétique comparative. Cette précaution ne remplace pas celles préconisées pour toute introduction d'espèce exotique (étude d'impact préalable dans les domaines écologique, sanitaire et génétique). Elle se surajoute dans les cas où on introduit une population issue d'un premier bassin hydrographique dans un second. 


\section{CONCLUSION}

Les connaissances nécessaires pour pouvoir introduire sans risque un taxon aquatique sur le sol national, ou d'un cours d'eau à l'autre, sont très loin d'être disponibles. Il est certain que nous ne disposerons jamais de toutes ces connaissances sur tous les taxons susceptibles de constituer un enjeu. II est donc clair que les quelques cas étudiés doivent servir de modèle à extrapoler. II en est des risques génétiques, comme des risques écologiques et sanitaires. C'est la raison pour laquelle ce texte essayant de définir les impacts génétiques des introductions est soutenu par de multiples exemples français (pour faire le point des connaissances) et étrangers (car tous les cas potentiels ne sont pas représentés en France).

La connaissance préalable de la structure génétique des taxons manipulés est nécessaire, car l'hybridation introgressive est reconnue comme un des risques majeurs consécutifs aux introductions (CRIVELLI, 1995; CRIVELLI et MAITLAND, 1995). Pour de nombreuses espèces qui ont pu faire l'objet d'analyses, des entités infraspécifiques, parfois fortement différentes par leur biologie et leur compatibilité, ont été mises en évidence. Quelques fois même, de vraies espèces sont découvertes ou réhabilitées, même en Europe (BERREBI et al., 1994), ce qui revêt une grande importance pour la protection de la biodiversité.

Quelques genres de salmonidés (GUYOMARD, 1989), cyprinidés (BERREBI, 1995c), anoures (NEVEU, 1989) ou chéloniens (FRITZ, 1995) ont montré la complexité de leur structuration systématique à travers l'Europe Occidentale, aux niveaux spécifique et infraspécifique. L'existence de telles structurations, démontrées pour certains taxons, est donc probable chez beaucoup d'autres, dont celles susceptibles d'être introduites ou manipulées.

Nous avons vu que les cas décrits d'introgression génétique les plus nombreux concernent des niveaux infraspécifiques. C'est souvent le cas lors des hybridations entre poissons. Elles sont souvent dues à des introductions, mais surtout à des transplantations de poissons d'un bassin à l'autre, ou pire d'une région écologique à l'autre dans un même pays (MACDONALD et al., 1989). Cela a été particulièrement observé en Italie (BIANCO, 1995), en Afrique du Sud (BRUTON et van AS, 1986) ou aux États-Unis. Une grande partie de ces hybridations passe inaperçue auprès des gestionnaires. Or, si le risque d'introduction de sousespèces étrangères susceptibles de s'hybrider avec des sous-espèces françaises est important, ce risque, lors de transfert à l'intérieur du territoire national d'une sous-espèce (ou simplement d'une forme différenciée) dans l'aire d'une autre sous-espèce, est encore plus grand (voir revue dans MINCKLEY, 1995). Selon MEFFE (1987), la protection devrait prendre en compte tous les niveaux : les espèces, sous-espèces, populations et même allèles uniques.

Devant ces risques, il faut donc souvent agir dans l'ignorance du cas précis mais dans la connaissance de quelques cas exemplaires. Ceci a mené à la proposition d'un " principe de précaution " adapté à la flore et à la faune des cours d'eau, particulièrement quand il s'agit de transplantations de populations : la prise en compte du bassin hydrographique comme unité structurelle a priori. Cette précaution doit se comprendre comme complémentaire aux études d'impact préalables à toute introduction.

\section{REMERCIEMENTS}

Ce travail de synthèse a été possible grâce à l'aide de nombreux collègues qui sont intervenus à divers stades du manuscrit. Mes remerciements vont particulièrement à $A$. CRIVELLI, G. FONTENELLE, R. GUYOMARD, E. PATTEE, H. PERSAT, E. ROSECCHI. Merci également à ceux qui m'ont rapidement fourni des références bibliographiques utiles tels M. ARTOIS, J. HAURY, P.J. LAURENT, H. MAURIN, F. MOUTOU, C. PERENNOU, A.M. PLANTY-TABACCHI et P. PONCIN. Un grand merci enfin à F. BERGOT pour son travail de coordination. 


\section{BIBLIOGRAPHIE}

ALLENDORF F.W., ESPELAND D.M., SCOW D.T., PHELPS S., 1980. Coexistence of native and introduced rainbow trout in the Kootenai River drainage. Proceedings of the Montana Academy of Sciences, 39, 28-36.

ALLENDORF F.W., LEARY R.F., 1988. Conservation and distribution of genetic variation in a polytypic species, the cutthroat trout. Conservation Biology, 2, 170-184.

ARTOIS M., MONTGELARD C., BAUTZ A.M., 1995. How to recognise mink : towards a biological test? (Preliminary results). Small Carnivore Conservation, 13, 15-16.

BARBAT-LETERRIER A., GUYOMARD R., KRIEG F., 1989. Introgression between introduced domesticated strains and Mediterranean native populations of brown trout (Salmo trutta L.). Aquatic Living Resources, 2, 215-223.

BEALL E., MORAN P., PENDAS A., IZQUIERDO J., GARCIA VAZQUEZ E., 1997. L'hybridation dans les populations naturelles de salmonidés dans le Sud-Ouest de l'europe et en milieu expérimental. Bull. Fr. Pêche Piscic., 344-345.

BEAUDOU D., 1993. Impact des déversements de truites domestiques dans les populations naturelles de truites communes (Salmo trutta fario). Etude dynamique et génétique. Cas du bassin de l'Orb (Hérault). Thèse de l'Université Montpellier II.

BERREBI P., 1995a. Analyse génétique des truites de Slovénie. Rapport final, Université Montpellier II, Montpellier, $13 \mathrm{p}$.

BERREBI P., 1995b. Analyse génétique des truites fario des rivières méditerranéennes des Pyrénées françaises ; marqueurs enzymatiques. Rapport de synthèse 1995, Université Montpellier II, Montpellier, $17 \mathrm{p}$.

BERREBI P., 1995c. Speciation of the genus Barbus in the north Mediterranean basin : recent advances from biochemical genetics. Biological Conservation, 72, 237-249.

BERREBI P., DUPONT F., CATTANEO-BERREBI G., CRIVELLI A.J., 1989. An isozyme study of the natural cyprinid hybrid Alburnus alburnus $x$ Rutilus rubilio in Greece. Journal of Fish Biology, 34, 307-313.

BERREBI P., BEAUDOU D., 1991. La génétique des populations appliquée à la truite fario. In Journée technique internationale "Truite commune pyrénéenne ". Génétique et repeuplement, Communauté de Travail des Pyrénées, Oronoz (Navarre, Espagne), 5-19.

BERREBI P., TSINGENOPOULOS K., KARAKOUSIS Y., SLECHTOVA V., POUYAUD L., 1994. Do Barbus barbus and Barbus meridionalis species complexes exist? In VIII Congress Societas Europaea Ichthyologorum "Fishes and their environment ", University of Oviedo, CSIC, IEO, Oviedo, Espagne, 71.

BIANCO P.G., 1995. Mediterranean endemic freshwater fishes of Italy. Biological Conservation, 72, 159-170.

BROWN L.R., MOYLE P.B., YOSHIYAMA R.M., 1994. Historical decline and current status of coho salmon in California. North American Journal of Fisheries Management, 14 (2), 237-261.

BRUTON M.N., van AS J.G., 1986. Faunal invasions of aquatic ecosystems in Southern Africa, with suggestion for their management. In MACDONALD I.A.W., KRUGER F.J., FERRAR A.A. (eds.), The ecology and management of biological invasions in Southern Africa, Oxford University Press, Cape Town, 47-61.

BUDIHNA N., OCVIRK A., 1990. Breeding and restocking of salmonid fishes in Slovenia. Journal of Fish Biology, 37 (suppl. A), 239-240. 
BUSACK C.A., CURRENS K.P., 1995. Genetic risks and hazards in hatchery operations fundamental concepts and issues. American Fisheries Society Symposium, 15, 71-80.

CADE T.J., 1983. Hybridization and gene exchange among birds in relation to conservation. In SCHONEWALD-COX C.M., CHAMBERS S.M., MACBRYDE B., THOMAS W.L. (eds.), Genetics and conservation : a reference for managing wild animal and plant populations, Benjamin/Cumming, London, 288-309.

CASTELLI M., 1994. Study on sex determination in the common barbel (Barbus barbus L.) (Pisces, Cyprinidae) using gynogenesis. In BEAUMONT A.R. (ed.), Genetics and evolution of aquatic organisms, Chapman and Hall, London, 509-519.

CRIVELLI A.J., 1995. Are fish introductions a threat to endemic freshwater fishes in the Northern Mediterranean region? Biological Conservation, 72, 311-319.

CRIVELLI A.J., MAITLAND P.S., 1995. Future prospects for the freshwater fish fauna of the North Mediterranean region. Biological Conservation, 72, 335-337.

CROCHET P.A., DUBOIS A., OHLER A., TUNNER H., 1995. Rana (Pelophylax) ridibunda Pallas, 1771, Rana (Pelophylax) perezi Seoane, 1885 and their associated klepton (Amphibia, Anura) : morphological diagnosis and description of a new taxon. Bulletin du Muséum National d'Histoire Naturelle, 4e série, 17 (1-2), 11-30.

DUBOIS A., 1983. A propos de cuisses de grenouilles. Alytes, 2 (3), 69-111.

FONTENELLE G., 1994. A propos de " repeuplement " en saumons dans le Nord-Ouest des États-Unis : succès ou échec ? ou comment interpréter certains mythes américains. In VIGNEUX E. (ed.), Journées d'information "Génétique et repeuplement ", Conseil Supérieur de la Pêche, Le Paraclet, France.

FORBES S.H., ALLENDORF F.W., 1991. Associations between mitochondrial and nuclear genotypes in cutthroat trout hybrid swarms. Evolution, 45 (6), 1332-1349.

FRITZ U., 1995. Zur innerartlichen Variabilität von Emys orbicularis (Linnaeus, 1758). Taxonomie in Mittel-Westeuropa, auf Korsika, Sardinien, der Apenninen-Halbinsel und Sizilien und Unterartengruppen von Emys orbicularis (Reptilia : Testudines: Emydidae). Zool. Abh. Mus. Tierkd. Dresden, 48 (13), 185-242.

GIUFFRA E., GUYOMARD R., FORNERIS G., 1996. Phylogenetic relationships and introgression patterns between incipient parapatric species of Italian brown trout (Salmo trutta L. complex). Molecular Ecology, 5, 207-220.

GOODFELLOW W.L., HOCUTT C.H., MORGAN R.P., STAUFFER J.R., 1984. Biochemical assessment of the taxonomic status of "Rhinichthys bowesi " (Pisces: Cyprinidae). Copeia, 1984, 652-659.

GRAY A.J., 1986. Do invading species have definable genetic characteristics? Phil. Trans. R. Soc. Lond., B314, 655-674.

GUENEGOU M.C., CITHAREL J., LEVASSEUR J.E., 1988. The hybrid status of Spartina anglica (Poaceae). Enzymatic analysis of the species and of the presumed parents. Canadian Journal of Botany, 66, 1830-1833.

GUENEGOU M.C., LEVASSEUR J., 1989. Le genre Spartina Schreb. dans le Massif Armoricain. In Colloques phytosociologiques XVIII “ Phytosociologie littorale et taxonomie ", Bailleul, France, 223-226.

GUYOMARD R., 1989. Diversité génétique de la truite commune. Bull. Fr. Pêche Piscic., 314, 118-135.

GUYOMARD R., 1997. Conséquences génétiques des introductions d'espèces de poissons en l'absence d'isolement reproducteur : intérêt et limites d'une approche en milieu expérimental. Bull. Fr. Pêche Piscic., 344-345. 
GYLLENSTEN U., LEARY R.F., ALLENDORF F.W., WILSON A.C., 1985. Introgression between two cutthroat trout subspecies with substantial karyotypic, nuclear, and mitochondrial genomic divergence. Genetics, 111, 905-915.

HUBBS C.L., 1955. Hybridization between fish species in nature. Systematic Zoology, 4, 1-20.

HUGHES B., in press. The ruddy duck Oxyura jamaicensis in Europe and the threat to the whiteheaded duck Oxyura leucocephala. In IWRB Symposium "Anatidae 2000 ", Strasbourg, France.

HUGHES B., GRUSSU M., 1994. The ruddy duck (Oxyura jamaicencis) in the United Kingdom : distribution, monitoring, current research and implications for European colonisation. Oxyura, 7, 29-47.

JESENSEK D., 1994. Artificial propagation of marble trout (Salmo marmoratus, Cuvier 1817) in the Fisheries Society of Tolmin. In KIRCHHOFER A., HEFTI D. (eds.), University of Bern, SSHL, FOEFL, Bern, Switzerland, 24 p.

JOHNSON K.R., WRIGHT J.E., 1986. Female brown trout $\times$ Atlantic salmon hybrids produce gynogens and triploids when backcrosses to reared Atlantic salmon. Aquaculture, 57, 345-358.

JOHNSON O.W., FLAGG T.A., MAYNARD D.L., MILNER G.B., WAKNITZ F.W., 1991. Status review of Lower Columbia River coho salmon. National Marine Fisheries Senvice, Seattle, Washington.

LARGIADĖR C.R., SCHOLL A., 1995. Effects of stocking on the genetic diversity of brown trout populations of the Adriatic and Danubian drainages in Switzerland. Journal of Fish Biology, 47 (suppl. A), 209-225.

LEARY R.F., ALLENDORF F.W., PHELPS S.R., KNUDSEN K.L., 1984. Introgression between westslope cutthroat and rainbow trout in the Clark Fork River drainage, Montana. Proceedings of the Montana Academy of Sciences, 43, 1-18.

LEARY R.F., ALLENDORF F.W., SAGE G.K., 1995. Hybridization and introgression between introduced and native fish. American Fisheries Society Symposium, 15, 91-101.

LIKNES G.A., GRAHAM P.J., 1988. Westslope cutthroat trout in Montana : life history, status, and management. American Fisheries Society Symposium, 4, 53-60.

MACDONALD A.W., LOOPE L.L., USHER M.B., HAMANN O., 1989. Wildlife conservation and invasion of nature reserves by introduced species : a global perspective. In DRAKE J.A. and MOONEY H.A. (eds.), Biological invasions : a global perspective. SCOPE, John Wiley and Sons Ltd, 215-255.

MACGOWAN C., DAVIDSON W.S., 1992. Unidirectional natural hybridization between brown trout (Salmo trutta) and Atlantic salmon (S. salar) in Newfoundland. Canadian Journal of Fisheries and Aquatic Sciences, 49, 1953-1958.

MACHORDOM A., BERREBI P., DOADRIO I., 1990. Spanish barbel hybridization detected using enzymatic markers : Barbus meridionalis Risso x Barbus haasi Mertens (Osteichthyes, Cyprinidae). Aquatic Living Resources, 3, 295-303.

MAIZERET C., MAURIN H., MIGOT P., LAFONTAINE L., 1995. Etat d'avancement du programme d'étude de la répartition du vison d'Europe en France. Cahiers d'Ethologie, 15 (2-3-4), 419-424.

MARCHANT C.J., 1967. Evolution of Spartina (Gramineae). I. The history and morphology of the genus in Britain. Journal of the Linnean Society of London, Botany, 60, 1-24.

MARCHANT C.J., 1968. Evolution of Spartina (Gramineae). II. Chromosomes, basic relationships and the problem of Spartina $x$ townsendii egg. Journal of the Linnean Society of London, Botany, 60, 381-409. 
MAURIN H., 1997. L'homme et les mammifères de France métropolitaine : évolution historique et introductions d'espèces dans les milieux humides et aquatiques. Bull. Fr. Pêche Piscic., 344-345.

MEFFE G.K., 1987. Conserving fish genomes : philosophies and practices. Environmental Biology of Fishes, 18, 3-9.

MINA M.V., 1991. Microevolution of fishes. In YABLOKOV A.V. (ed.), Evolutionary aspects of phenetic diversity. Amerind Publishing Co., Oxonian Press Pvt. Ltd, New Delhi, India, $215 \mathrm{p}$.

MINCKLEY W.L., 1995. Translocation as a tool for conserving imperiled fishes : experiences in Western United States. Biological Conservation, 72, 297-309.

MOBBERLEY D.G., 1956. Taxonomy and distribution of the genus Spartina. Journal of Sciences, 30, 471-574.

MORIZOT D.C., CALHOUN S.W., CLEPPER L.L., SCHMIDT M.E., WILLIAMSON J.H., CARMICHAEL G.J., 1991. Multispecies hybridization among native and introduced centrarchid basses in central Texas. Transactions of the American Fisheries Society, 120 , 283-289.

NEHLSEN W., WILLIAMS J.E., LICHATOWICH J.A., 1991. Pacific salmon at the crossroads: stocks at risk from California, Oregon, Idaho and Washington. Fisheries (Bethesda), 16 (2), 4-21.

NELSON K., SOULÉ M., 1987. Genetical conservation of exploited fishes. In RYMAN N., UTTER F. (eds.), Population Genetics and Fisheries Management, University of Washington Press, Seattle, 345-368.

NEVEU A., 1989. Rana ridibunda. In CASTANET J., GUYETANT R. (eds.), Atlas de répartition des Amphibiens et Reptiles de France. Société Herpétologique de France, Paris, 91 p.

NEVEU A., 1997. L'introduction d'espèces allochtones de grenouilles vertes en France, deux problèmes différents : celui de $R$. catesbeiana et celui des taxons non présents du complexe Esculenta. Bull. Fr. Pêche Piscic., 344-345.

NYGREN A., NYMAN L., SVENSSON K., JAHNKE G., 1975. Cytological and biochemical studies in backcrosses between the hybrid Atlantic salmon $\times$ sea trout and its parental species. Hereditas, 81, 55-62.

OCVIRK A., 1989. Study of the marble trout (Salmo marmoratus, Cuvier 1817) population in Slovenia from 1962 to 1988 (in serbo-croat). Ichthyos, 8, 37-59.

PERENNOU C., 1997. L'Érismature rousse (Oxyura jamaicensis) : une introduction problématique d'oiseau dans les milieux aquatiques. Bull. Fr. Pêche Piscic., 344-345.

PERSAT H., EPPE R., 1997. Alevinage, pollution et cloisonnement de l'espace fluvial dans les structures génétiques des populations de poisson : l'ombre commun, Thymallus thymallus, dans le Rhône au niveau de la Savoie. Bull. Fr. Pêche Piscic., 344-345.

PHILIPP D.P., 1991. Genetic implications of introducing Florida largemouth bass, Micropterus salmoides floridanus. Canadian Journal of Fisheries and Aquatic Sciences, 48 (suppl. 1), 58-65.

POTEAUX C., 1995. Interactions génétiques entre formes sauvages et formes domestiques chez la truite commune (Salmo trutta fario L.). Thèse de I'Université Montpellier II, $110 \mathrm{p}$.

POTEAUX C., BONHOMME F., BERREBI P., soumis. Microsatellite polymorphism and genetic impact of restocking in Mediterranean brown trout (Salmo trutta fario L.). Heredity. 
POTEAUX C., BERREBI P., 1997. Intégrité génomique et repeuplements chez la truite commune du versant méditerranéen. Bull. Fr. Pêche Piscic., 344-345.

POVZ M., 1989. Distribution and biometric characteristics of the marble trout (Salmo marmoratus Cuvier 1817) in Slovenia. Ichthyos, 8, 29-36.

POVZ M., 1995. Status of freshwater fishes in the Adriatic catchment of Slovenia. Biological Conservation, 72, 171-177.

POVZ M., JESENSEK D., BERREBI P., CRIVELLI A.J., 1996. The marble trout, Salmo trutta marmoratus, Cuvier 1817, in the Soca River basin, Slovenia. Tour du Valat Publications, $72 \mathrm{p}$.

ROSECCHI E., POIZAT G., CRIVELLI A.J., 1997. Introductions de poissons d'eau douce et d'écrevisses en Camargue : historique, origines et modifications des peuplements. Bull. Fr. Pêche Piscic., 344-345.

SCHULER J.F., 1954. Natural mutations in inbred lines of maize and their heterotic effect. I. Comparison of parent, mutant and their F1 hybrid in a highly inbred background. Genetics, 39, 908-922.

TEMPLETON A.R., 1986. Coadaptation and outbreeding depression. In SOULÉ M.E. (ed.), Conservation biology, the science of scarcity and diversity. Sinauer Associates, Sunderland, Massachussetts, 105-116.

URDIALES C., PREIRA P., 1993. Clés d'identification de O. jamaicensis, O. leucocephala et ses hybrides. ICONA, Madrid.

VERSPOOR C., HAMMAR J., 1991. Introgressive hybridization in fishes : the biological evidence. Journal of Fish Biology, 39 (suppl. A), 309-334.

WHITMORE D.H., 1983. Introgressive hybridization of smallmouth bass (Micropterus dolomieu) and Guadalupe bass ( $M$. treculi). Copeia, 1983, 672-679.

WILDE G.R., ECHELLE A.A., 1992. Genetic status of Pecos pupfish populations after establishment of a hybrid swarm involving an introduced congener. Transaction of the American Fisheries Society, 121, 277-286.

WOOD C.C., FOOTE C.J., 1990. Genetic differences in the early development and growth of sympatric sockeye salmon and kokanee Oncorhynchus nerka and their hybrids. Canadian Journal of Fisheries and Aquatic Sciences, 47, 2250-2260.

WRIGHT S., 1993. Fishery management of wild Pacific salmon stocks to prevent extinctions. Fisheries, 18 (5), 3-4. 